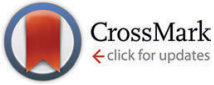

Cite this: J. Mater. Chem. C, 2015 3, 10907

Received 28th July 2015, Accepted 14th September 2015

DOI: $10.1039 / c 5 t c 02310 f$

www.rsc.org/MaterialsC

\section{Synthesis and super-resolution imaging performance of a refractive-index-controllable microsphere superlens $\dagger$}

\begin{abstract}
Haie Zhu, ${ }^{a}$ Bing Yan, ${ }^{b}$ Shuxue Zhou, ${ }^{a}$ Zengbo Wang ${ }^{*^{b}}$ and Limin $W^{*^{a}}$
Microspheres can function as optical superlenses for nanoscale super-resolution imaging. The imaging performance is mainly affected by the size and refractive index of the microsphere. Precise control of these parameters is a challenging task but of fundamental importance to the further development of the technique. In this study, we demonstrate for the first time a nanoparticle-hybrid suspension polymerization approach to chemically synthesize high-quality microspheres $\left(\mathrm{ZrO}_{2} /\right.$ polystyrene) with optical properties that are highly controllable. Microspheres of different sizes $(d: 2-20 \mu \mathrm{m})$ and refractive indexes $\left(n_{\mathrm{p}}: 1.590-1.685\right)$ were synthesized and their super-resolution imaging performances were evaluated and compared. Our results show that continuously increasing the refractive index of microspheres can enhance the imaging resolution and quality. A $60 \mathrm{~nm}$ resolution has been obtained in the wide-field imaging mode and a $50 \mathrm{~nm}$ resolution has been obtained in the confocal mode imaging of semiconductor chip samples. The obtained 50-60 nm resolutions have significantly gone beyond the conventional $200 \mathrm{~nm}$ resolution limit for visible light optical microscopes; the super-resolution mechanism has been discussed. The synthesized microsphere superlenses may find applications in many other areas as well, including nanolithography, nanosensing, nano-diagnosis, nano-spectroscopy and ultra-high density optical data storage.
\end{abstract}

\section{Introduction}

Optical microscopes are indispensable tools for studying microscale features, especially for imaging of live biological samples. Compared with electron microscopy, optical microscopy is noninvasive and does not require a high vacuum. However, optical microscopy resolution is often limited at $200 \mathrm{~nm}$, which is considerably inferior compared to the resolution of electron microscopes of about $10 \mathrm{~nm}$. The limited optical resolution, known as the Abbe diffraction limit, ${ }^{1}$ stems from the wave nature of light and is caused by the loss of evanescent waves in the far-field, which contains high spatial frequency subwavelength information of an object. ${ }^{2}$ It shall be noted, however, the recent Nobel Prize awarded to super-resolution fluorescence microscopy techniques do not rely on the recovery of evanescent waves in the far-field. These techniques use strategies of patterned light excitation or single molecule localization of fluorescent molecules. ${ }^{3}$ In other words, the improvement of resolution in

\footnotetext{
${ }^{a}$ Department of Materials Science and State Key Laboratory of Molecular

Engineering of Polymers, Fudan University, Shanghai 200433, China.

E-mail:Imw@fudan.edu.cn

${ }^{b}$ School of Electronic Engineering, Bangor University, Bangor LL57 1UT, UK.

E-mail:z.wang@bangor.ac.uk

$\dagger$ Electronic supplementary information (ESI) available. See DOI: 10.1039/ c5tc02310f
}

fluorescence super-resolution techniques is achieved through material response instead of optical system itself. Other superresolution techniques, such as metamaterials superlenses, ${ }^{4}$ super-oscillatory lenses, ${ }^{5}$ plasmon gratings, ${ }^{6}$ and nanoscale solid immersion lenses (SILs), ${ }^{7-9}$ have the root connection with the transformation of evanescent waves into propagating waves. These techniques, however, have one drawback or another in optical super-resolution imaging. For example, metamaterial lenses, super-oscillatory lenses and plasmon gratings are very difficult to manufacture and can operate only in a narrow range of wavelengths. SILs operate by coupling of hemispherical or super-hemispherical dielectric lenses or microlens arrays with optical microscopy. The SILs could be fabricated by a selfassembly method, ${ }^{8}$ thermal reshaping method ${ }^{9}$ or the "topdown" fabrication approaches such as femtosecond laser direct writing, and highly ordered three dimensional microlens arrays even with dynamically tunable biopolymers have already been fabricated. ${ }^{10,11}$ However, it still remains a great challenge to resolve structures below $100 \mathrm{~nm}$ due to the low refractive index $(<1.6)$ of the lens materials or the nanoscale separation between the surfaces of the SILs and the investigated substrates.

Recently, super-resolution imaging through coupling of dielectric microspheres with white-light optical microscopy have proven to be a simple and superior way to achieve sub-diffraction resolution. ${ }^{2,12-17}$ Compared with SILs, the contact region for a 
hard microsphere is a single point, which is significantly smaller, thus making sure that the lower surface of microspheres is located within the extent of evanescent field produced by the underlying nano-patterned substrate. Thus, the microsphere can convert the high frequency spatial harmonics of the evanescent field into propagating waves to form a magnified virtual image below the substrate surface, which can be picked up by a conventional objective lens. Initially, this technique had been developed for commercial $\mathrm{SiO}_{2}$ spheres submerged in an air environment, ${ }^{2}$ achieving $50 \mathrm{~nm}$ resolution of a gold-coated anodic alumina oxide membrane. However, due to the limitation of the low refractive index of $\mathrm{SiO}_{2}$ spheres, the super-resolution window of $\mathrm{SiO}_{2}$ spheres was confined to the diameter $(D)$ range of 2-9 $\mu \mathrm{m}$. Silica spheres with diameters larger than $10 \mu \mathrm{m}$ even failed in $100 \mathrm{~nm}$ resolution imaging tests. Large diameter $(30-100 \mu \mathrm{m})$ polystyrene microspheres (refractive index of the particle: $n_{\mathrm{p}}=\sim 1.59$ ) were also reported for the super-resolution imaging of a blue-ray disc in an air atmosphere, achieving a resolution of $120 \mathrm{~nm} .{ }^{11}$ However, in these cases, the microspheres exposed in an air environment usually resulted in a low imaging contrast. When $\mathrm{SiO}_{2}$ spheres were semi-immersed in a thin layer of ethanol or SU-8 resist, ${ }^{15,16}$ the imaging contrast could be strongly strengthened; however, it was not easy to resolve features below $100 \mathrm{~nm}$ because of their low refractive index $\left(n_{\mathrm{p}}=\sim 1.46\right)$. Darafsheh et al. found that when $\mathrm{BaTiO}_{3}\left(n_{\mathrm{p}}=1.9\right)$ glass spheres were totally submerged in a liquid of isopropyl alcohol, ${ }^{12}$ the small spheres $($ diameter $=4.2 \mu \mathrm{m}$ ) were able to discern features as small as $75 \mathrm{~nm}(\sim \lambda / 7)$, while for large microspheres $(50 \mu \mathrm{m}<$ diameter $<220 \mu \mathrm{m})$, the resolved feature sizes were only $\sim \lambda / 4$. Li et al. reported water submerged $\mathrm{BaTiO}_{3}$ (diameter $=100 \mu \mathrm{m}$ ) for the imaging of an adenovirus with a resolution of $75 \mathrm{~nm}^{14}$

To date, microspheres coupled to optical super-resolution imaging were focused on commercial $\mathrm{SiO}_{2}$, PS and high $\mathrm{RI} \mathrm{BaTiO}_{3}$. However, these spheres have either a low refractive index or poor processability, which make them little less attractive in practical applications. Introducing high RI inorganic nanoparticles into a polymer matrix may be a promising strategy to obtain high refractive index nanocomposites, which combine the advantages of an organic matrix (low weight, good impact resistance and excellent processability) and inorganic materials (high RI and good thermal stability). ${ }^{18}$ In fact, the high RI nanocomposite films ${ }^{19-21}$ or bulk nanocomposites ${ }^{22}$ have already been reported by some researchers. However, due to the arguable difficulty, the fabrication of high RI nanocomposite microspheres with high optical transparency for the super-resolution imaging has barely been reported to the best of our knowledge. Herein, we report a facial suspension polymerization method for the preparation of optically transparent and high $\mathrm{RI} \mathrm{ZrO}_{2} / \mathrm{PS}$ nanohybrid colloidal microspheres. Compared with the femtosecond laser direct writing method, which is usually applied to the fabrication of lenses composed of low refractive index photosensitive molecules, the suspension polymerization method used here can fabricate spheres with high and controllable refractive index. Herein, as high as $50.8 \mathrm{wt} \%$ of $\mathrm{ZrO}_{2}$ nanoparticles can be uniformly distributed in the final composite microspheres, which makes these colloidal microspheres have a controllable refractive index $\left(n_{\mathrm{p}}=1.590-1.685\right)$. These microspheres are used for optical super-resolution imaging and features as small as 50-60 $\mathrm{nm}$ are clearly resolved.

\section{Experimental}

\section{Materials}

Zirconium(Iv) isopropoxide-isopropanol complex (99.9\%) was purchased from Aldrich. Anhydrous benzyl alcohol (99.8\%), 3-(trimethoxysilyl) propyl methacrylate (MPS, 97\%), styrene (S, 99\%), oleic acid (OA), lauroyl peroxide (98\%), divinylbenzene (DVB, 80\%), and cedarwood-oil were purchased from Aladdin Reagent Co., Ltd (China). Polyvinyl alcohol (PVA1788), tetrahydrofuran (THF, $\geq 99.5 \%$ ), anhydrous methanol $(\geq 99.5 \%)$, anhydrous ethanol ( $\geq 99.7 \%$ ), and dimethyl-silicone were obtained from Sinopharm Chemical Reagent Corp (China). Styrene was distilled under reduced pressure to remove the inhibitor hydroquinone, which was then stored in a refrigerator at $4{ }^{\circ} \mathrm{C}$. Other reagents were used as received.

\section{Synthesis of $\mathrm{ZrO}_{2}$ nanoparticles}

$\mathrm{ZrO}_{2}$ nanoparticles were synthesized according to a method described elsewhere. ${ }^{23}$ Briefly, zirconium(Iv) isopropoxideisopropanol complex (6.25 g) and benzyl alcohol $(60 \mathrm{~mL})$ were charged into a $100 \mathrm{~mL}$ Teflon-lined autoclave. This Teflon-lined autoclave was sealed and placed into an oven at a temperature of $240{ }^{\circ} \mathrm{C}$. After 4 days, the reaction mixture was allowed to cool down, and a white turbid suspension was obtained.

\section{Modification of $\mathrm{ZrO}_{2}$ nanoparticles}

The as-synthesized $\mathrm{ZrO}_{2}$ nanoparticle suspension was centrifuged to obtain a white precipitate and then washed by three cycles of sonication and centrifugation with ethanol. The wet $\mathrm{ZrO}_{2}$ nanoparticles were dispersed in the pre-made THF/MPS solution based on a molar ratio of $0.4 / 1$ for MPS-to- $\mathrm{ZrO}_{2}$ and $1.22 \mathrm{wt} \%$ of $\mathrm{ZrO}_{2}$. The dispersion was sonicated for $30 \mathrm{~min}$ at room temperature, and then oleic acid $(\mathrm{OA}, 500 \mu \mathrm{L})$ was added and the mixture was sonicated for another $30 \mathrm{~min}$. The transparent dispersion was placed into an oven at a temperature of $60{ }^{\circ} \mathrm{C}$ for $20 \mathrm{~h}$ and at $80^{\circ} \mathrm{C}$ for another $3 \mathrm{~h}$. The dispersion was centrifuged at $7000 \mathrm{rpm}$ for $10 \mathrm{~min}$ to remove the possible aggregates and then concentrated by a rotary evaporator to remove most of the THF. The concentrated MPS and oleic acid modified $\mathrm{ZrO}_{2}$ nanoparticles slurry was precipitated and washed by two cycles of sonication and centrifugation in ethanol. The purified $\mathrm{MPS} / \mathrm{OA} / \mathrm{ZrO}_{2}$ nanoparticles were added into a styrene monomer to obtain a yellowish transparent solution upon ultrasonication, which was then stored in a refrigerator.

\section{Preparation of $\mathrm{ZrO}_{2} / \mathrm{PS}$ nanohybrid colloidal microspheres}

Suspension polymerization was used to synthesize the $\mathrm{ZrO}_{2} / \mathrm{PS}$ nanohybrid colloidal microspheres. In detail, polyvinyl alcohol was added into deionized water and heated at $60{ }^{\circ} \mathrm{C}$ for $20 \mathrm{~min}$ to obtain a $0.175 \mathrm{wt} \%$ of PVA aqueous solution, and then it was allowed to cool. Various amounts of $\mathrm{MPS} / \mathrm{OA} / \mathrm{ZrO}_{2}$ nanoparticles 
dispersed in styrene, $10 \mathrm{wt} \%$ of DVB in terms of S and $4 \mathrm{wt} \%$ of lauroyl peroxide in terms of $\mathrm{S}$ as the initiator were mixed and then poured into a PVA aqueous solution, and emulsified with a homogenizer at $6000 \mathrm{rpm}$ for $3 \mathrm{~min}$. The emulsified dispersion was then poured into a $250 \mathrm{~mL}$ four-neck round-bottom flask equipped with a mechanical stirrer, a reflux condenser, a thermocouple, and a nitrogen gas inlet. The system was degassed with nitrogen for $30 \mathrm{~min}$ to remove oxygen in the reactor, which was heated to $70{ }^{\circ} \mathrm{C}$ for polymerization at $250 \mathrm{rpm}$ for $12 \mathrm{~h}$ under a nitrogen atmosphere. The products were labelled as M1, M2, M3, M4, and M5, corresponding to 10.5, 23.5, 30.3, 38.2, and $50.8 \mathrm{wt} \%$ of $\mathrm{ZrO}_{2}$ incorporated microspheres based on TGA analysis. As a control, bare polymer microsphere M0 was prepared in the same way but without $\mathrm{ZrO}_{2}$ nanoparticles. The as-synthesized microspheres were obtained by centrifugation and washing by three cycles of sonication and centrifugation with ethanol, and dried under vacuum for characterization.

\section{Super-resolution imaging by composite microspheres}

To demonstrate the super-resolution property of the microspheres, the as-synthesized nanohybrid colloidal microspheres were observed with an Olympus optical microscope (BX63) under an objective lens of $100 \times$ with a numerical aperture of 0.8 and reflective mode illumination with a halogen light source for the optical imaging. The sub-diffraction-limited objects used in the experiment were semiconductor chips with nano-patterned structures of $75 \mathrm{~nm}$ and $60 \mathrm{~nm}$ gaps between specific stripes with widths of $145 \mathrm{~nm}$ and $115 \mathrm{~nm}$, respectively. For imaging the $75 \mathrm{~nm}$ gaps, the microspheres were first spread on the surfaces of the sub-diffraction-limited objects through the evaporation of ethanol drops; subsequently, dimethyl-silicone (refractive index of the medium is $n_{\mathrm{m}}=1.400$ ) was dropped onto the substrates to make the microspheres be semi-immersed in silicon oil after spin-coating at a speed of $3000 \mathrm{rpm}$ for $5 \mathrm{~min}$. For imaging the $60 \mathrm{~nm}$ gaps, the microspheres were semi-immersed in cedarwood oil $\left(n_{\mathrm{m}}=1.515\right)$ through spin-coating in the same way as dimethyl-silicone; then, most of the cedarwood-oil was washed away by tetrachloroethylene to reduce the accumulation of oil around the spheres. The sub-diffraction virtual images of the nano-patterned surfaces were acquired by focusing through the microspheres into the substrates. In addition, more complicated structures of the chips with $50 \mathrm{~nm}$ gaps were also imaged by coupling the dimethyl-silicone semi-immersed microspheres with an Olympus LEXT OLS4100 scanning laser confocal microscope under reflection mode laser illumination at $\lambda=405 \mathrm{~nm}$. Super-resolution images were acquired by a $100 \times$ and NA $=0.95$ objective lens.

\section{Characterization}

Thermogravimetric analysis (TGA) was carried out using a Perkin Elmer TGA-7 instrument (USA) at a heating rate of $10{ }^{\circ} \mathrm{C} \mathrm{min}^{-1}$ from room temperature to $800{ }^{\circ} \mathrm{C}$ in air. Fourier transform infrared spectra (FTIR) were acquired with a Nicolet Nexus 470 spectrometer (USA). High resolution transmission electron microscopy (HRTEM) was performed on a Tecnai G2 F20 $\mathrm{S}$-Twin field emission transmission electron microscope (USA).
The $\mathrm{ZrO}_{2} / \mathrm{PS}$ nanohybrid colloidal microspheres were embedded in epoxy resin and ultramicrotomed into $70 \mathrm{~nm}$ thick slices for HRTEM measurements. The morphologies of the microspheres were characterized by a Philips XL30 scanning electron microscope (SEM). The chemical composition and distribution of the composite microspheres were characterized by a Zeiss Ultra 55 field-emission scanning electron microscope (Germany) equipped with an X-ray energy dispersive spectroscope (EDS) for elemental area analysis (SEM mapping) and elemental line analysis (SEM linescan). The geometry profiles of the oil immersed in the microsphere lenses were characterized by the three-dimensional image acquired from an Olympus LEXT OLS4100 scanning laser confocal microscope, which showed the distribution of oil around the sphere.

\section{Results and discussion}

\section{Synthesis of $\mathrm{ZrO}_{2} / \mathrm{PS}$ nanohybrid colloidal microspheres}

The nonaqueous synthesis of zirconium(Iv) isopropoxide in benzyl alcohol produced a white precipitate of $\mathrm{ZrO}_{2}$. To prepare a transparent dispersion of $\mathrm{ZrO}_{2}$ in an organic monomer, MPS was first chemically bonded to the $\mathrm{ZrO}_{2}$ nanoparticles through condensation between the alkoxyl groups of MPS and the surface hydroxyl groups of the $\mathrm{ZrO}_{2}$. MPS provides not only steric stabilization of the $\mathrm{ZrO}_{2}$ nanoparticles in organic monomer but also a double bond for the subsequent polymerization. However, in the experiment, if only MPS was used as the ligand, the MPS-modified $\mathrm{ZrO}_{2}\left(\mathrm{MPS} / \mathrm{ZrO}_{2}\right)$ nanoparticles were well dispersed in styrene, but tended to migrate from the interior of the monomer drops to their surfaces when the monomer droplets containing $\mathrm{MPS} / \mathrm{ZrO}_{2}$ were suspended in a PVA aqueous solution and polymerized through suspension polymerization, resulting in collapsed spheres. This behavior may be attributed to the lack of hydrophobicity of the MPS molecules. To improve the hydrophobicity of the $\mathrm{MPS} / \mathrm{ZrO}_{2}$ nanoparticles, OA with a longer hydrophobic alkyl chain was used as the second ligand, which could effectively anchor to the $\mathrm{ZrO}_{2}$ surface through coordination between its carboxyl group and the $\mathrm{Zr}$ atom. ${ }^{23}$ The MPS/OA/ZrO $\mathrm{Zr}_{2}$ can not only be dispersed in the monomers of $S$ and DVB in a high concentration, but also polymerize with them by suspension polymerization, forming nearly perfect $\mathrm{ZrO}_{2} / \mathrm{PS}$ composite microspheres with $\mathrm{ZrO}_{2}$ nanoparticles evenly distributed within the microspheres. A small amount of DVB was used as the crosslinking agent to accelerate the polymerization rate and form the cross-linked network structure, which would further prevent the possible movement of $\mathrm{ZrO}_{2}$ nanoparticles towards the surfaces of spheres during suspension polymerization. This synthesis process is highly repeatable and designable. The most critical step is the modification of $\mathrm{ZrO}_{2} \cdot{ }^{24,25}$ Once the well modified $\mathrm{ZrO}_{2}$ nanoparticles are prepared, they can be dispersed in monomer such as styrene in various amounts (0-50.8 wt\%), thus the refractive index of the $\mathrm{ZrO}_{2} /$ PS nanohybrid colloidal microspheres can be designed by the $\mathrm{ZrO}_{2}$ content. 


\section{Morphology and structure of the $\mathrm{ZrO}_{2}$ nanocrystals and $\mathrm{ZrO}_{2} / \mathrm{PS}$ nanohybrid colloidal microspheres}

Fig. 1 demonstrates the typical TEM images of the MPS/OA/ $\mathrm{ZrO}_{2}$ nanoparticles. The sample was dispersed well in THF with a uniform crystal size of about $3.5 \mathrm{~nm}$ (Fig. 1a). The high crystalline lattice of the nanoparticles, as shown in Fig. 1b, belongs to the cubic phase (JCPDS: 27-991). ${ }^{23}$

The FTIR spectrum of MPS/OA/ $\mathrm{ZrO}_{2}$ nanoparticles shows the absorption peaks at $500-700 \mathrm{~cm}^{-1}$ and $800-1100 \mathrm{~cm}^{-1}$ assigned to $\mathrm{Zr}-\mathrm{O}$ and $\mathrm{Si}-\mathrm{O}-\mathrm{Zr}$ vibrations (Fig. 2a), respectively, indicating that MPS has been chemically bonded to the $\mathrm{ZrO}_{2}$ nanoparticles. The peaks at 1547 and $1460 \mathrm{~cm}^{-1}$ belong to the characteristic absorption of $-\mathrm{COO}^{-}$groups, meaning that OA has been chemically absorbed to the surface of $\mathrm{ZrO}_{2}$ nanoparticles as well. The absorption peaks at $1719 \mathrm{~cm}^{-1}, 2923 \mathrm{~cm}^{-1}$ and $2852 \mathrm{~cm}^{-1}$ are attributed to carbonyl vibration, $\mathrm{C}-\mathrm{H}$ vibration in $-\mathrm{CH}_{3}$ and $-\mathrm{CH}_{2}$, respectively. All these results unveil the successful modification of both MPS and OA on the surface of the $\mathrm{ZrO}_{2}$ nanoparticles. The FTIR spectrum of $\mathrm{ZrO}_{2}$ /PS nanohybrid microspheres (Fig. 2b) shows absorption peaks at $500-700 \mathrm{~cm}^{-1}$ for the $\mathrm{Zr}-\mathrm{O}$ vibration, at $1451 \mathrm{~cm}^{-1}$ and $1492 \mathrm{~cm}^{-1}$ for the characteristic peaks of phenyl stretching vibration and at $757 \mathrm{~cm}^{-1}$ and $700 \mathrm{~cm}^{-1}$ for flexural vibration $\left(\delta_{\mathrm{C}-\mathrm{H}}\right)$ of the benzene ring, which demonstrates the successful encapsulation of $\mathrm{ZrO}_{2}$ in the hybrid microspheres.

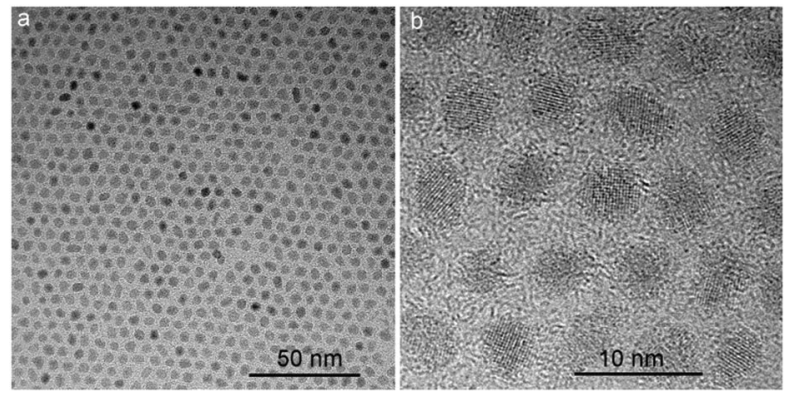

Fig. 1 ( $a$ and b) TEM images of MPS/OA/ZrO 2 nanoparticle under low and high magnification.

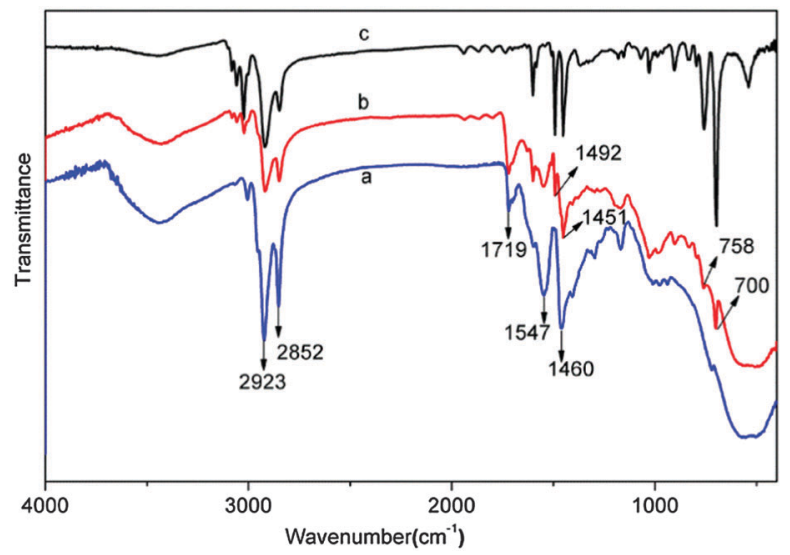

Fig. 2 FTIR spectrum of (a) MPS/OA/ $\mathrm{ZrO}_{2}$ nanoparticles, (b) $\mathrm{ZrO}_{2} / \mathrm{PS}$ nanohybrid colloidal microspheres and (c) pure PS colloidal microspheres.

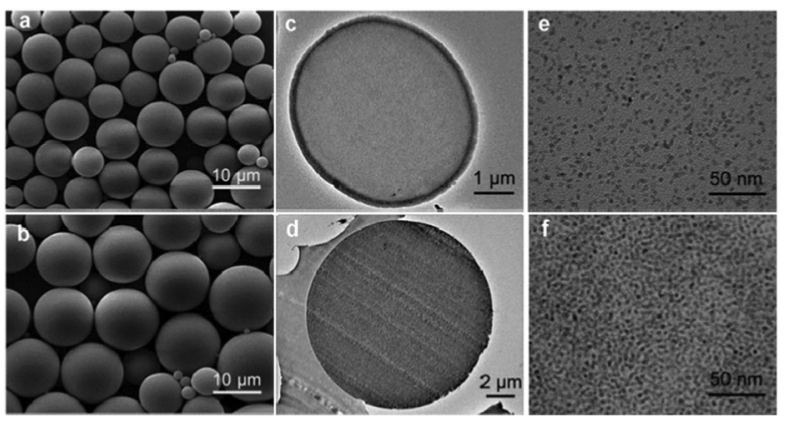

Fig. 3 SEM images of $\mathrm{ZrO}_{2} / \mathrm{PS}$ nanohybrid colloidal microspheres: (a) M1 and (b) M5; TEM images of ultrathin sections of M1 ( $c$ and e) and M5 (d and f).

Fig. 3a and $\mathrm{b}$ present the typical SEM images of the $\mathrm{ZrO}_{2} / \mathrm{PS}$ nanohybrid colloidal microspheres M1 and M5, which have a smooth spherical surface morphology. All the samples from M1 to M5 have smooth surfaces even at a high $\mathrm{ZrO}_{2}$ content of $50.8 \mathrm{wt} \%$, which can be further seen from their high magnification SEM images (see Fig. S1, ESI $\dagger$ ). The TEM images of the ultrathin sections, as shown in Fig. 3c-f, further display that $\mathrm{ZrO}_{2}$ nanoparticles are uniformly distributed at their primary size $(\sim 3.5 \mathrm{~nm})$ in the polymer matrix, even at as high as $50.8 \mathrm{wt} \%$ of $\mathrm{ZrO}_{2}$ content. This can ensure that the nanohybrid microspheres are highly transparent to visible light, which is necessary for microsphere superlenses to improve the resolution of optical microscopy.

Typical SEM mapping graphs of the nanohybrid colloidal microsphere clearly reveal the existence of $\mathrm{Zr}, \mathrm{C}$, and $\mathrm{O}$ elements in hybrid microspheres (Fig. 4a-d), with well-defined spherical structure. A SEM linescan of the individual microsphere further demonstrates the uniform distribution of $\mathrm{Zr}$ in the microsphere (Fig. 4e). From the corresponding linescan spectrum in Fig. 4f, the relative weight contents of $\mathrm{Zr}, \mathrm{C}$, and $\mathrm{O}$ are found to be $36.0 \%, 49.6 \%$, and $14.4 \%$, respectively. The composition of samples M1-M4 were also analysed by SEM linescan analysis; all the samples show uniform distribution of $\mathrm{Zr}, \mathrm{C}$, and $\mathrm{O}$ in the hybrid microspheres, and their relative weight contents of $\mathrm{Zr}, \mathrm{O}$, and $\mathrm{C}$ are summarized in Table 1.

Fig. 5 shows the thermogravimetric analysis (TGA) results of the modified $\mathrm{ZrO}_{2}$ nanoparticles and $\mathrm{ZrO}_{2} / \mathrm{PS}$ composite
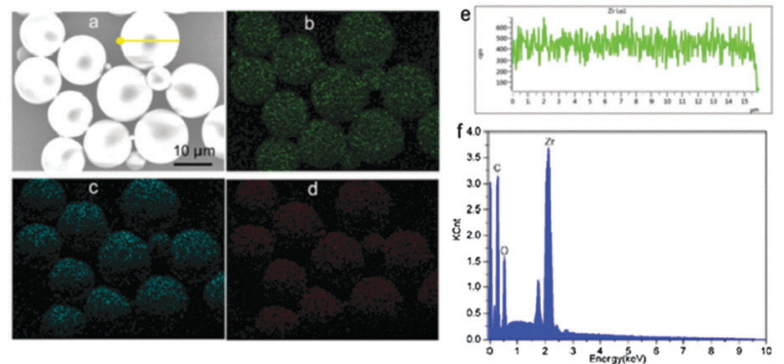

Fig. 4 SEM image (a) and SEM mapping photographs of $\mathrm{Zr}$ (b), C (c) and O (d) in the nanohybrid microspheres M5; a yellow line is stretched across a selected microsphere in the top left SEM image for the SEM linescan element analysis. (e) SEM linescan element distribution of $Z r$ in the selected microsphere of M5. (f) The corresponding linescan EDX spectrum of the microsphere. 
Table 1 SEM linescan results of $\mathrm{Zr}, \mathrm{O}$, and $\mathrm{C}$ content in M1-M5

\begin{tabular}{lccc}
\hline Sample code & Zr $(\mathrm{wt} \%)$ & O (wt\%) & C (wt\%) \\
\hline M1 & 5.79 & 4.64 & 89.6 \\
M2 & 14.9 & 6.88 & 78.2 \\
M3 & 19.3 & 10.1 & 70.6 \\
M4 & 27.4 & 11.6 & 61.1 \\
M5 & 36.0 & 14.4 & 49.6 \\
\hline
\end{tabular}

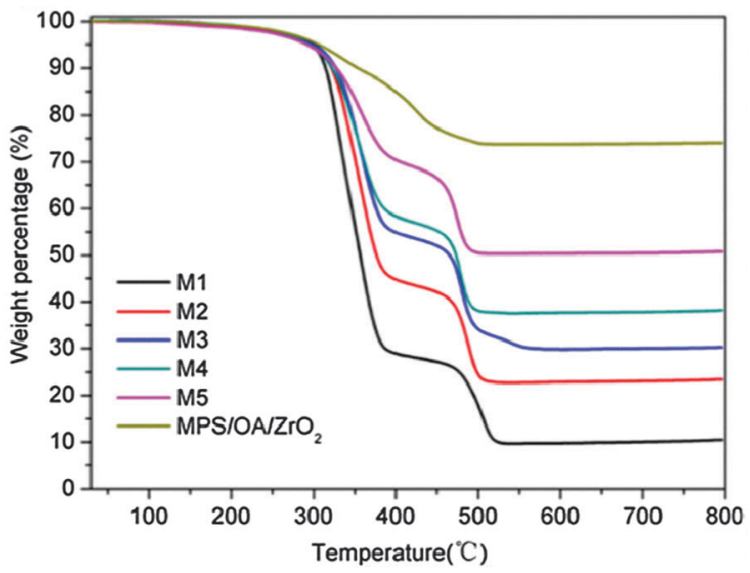

Fig. 5 TGA curves of MPS/OA/ZrO ${ }_{2}$ nanoparticles and $\mathrm{ZrO}_{2} / \mathrm{PS}$ nanohybrid colloidal microspheres M1-M5.

microspheres M1-M5. The weight loss of the modified nanoparticles below $200{ }^{\circ} \mathrm{C}$ is $1 \mathrm{wt} \%$, which is attributed to the loss of physically absorbed water and organic solvent. The weight loss between 200 and $800{ }^{\circ} \mathrm{C}$ is $25 \mathrm{wt} \%$, corresponding to the chemically bonded OA and MPS on the $\mathrm{ZrO}_{2}$ nanoparticles. The residual inorganic substances are about 10.5, 23.5, 30.3, 38.2 and $50.8 \mathrm{wt} \%$ for samples M1, M2, M3, M4, and M5, respectively, and are mainly composed of $\mathrm{ZrO}_{2}$.

The refractive index of M0-M5 could be theoretically estimated according to the following formula:

$$
n_{\mathrm{p}}=n_{\mathrm{org}} \times V_{\mathrm{org}}+n_{\mathrm{ZrO}_{2}} \times V_{\mathrm{ZrO}_{2}}
$$

where $n_{\mathrm{org}}$ and $n_{\mathrm{ZrO}}$ are the refractive indices of polymer $\left(n_{\text {org }} \approx 1.59\right)$ and $\mathrm{ZrO}_{2}$ nanoparticles $\left(n_{\mathrm{ZrO}_{2}} \approx 2.2\right) . V_{\text {org }}$ and $V_{\mathrm{ZrO}_{2}}$ are the volume fractions of polymer and $\mathrm{ZrO}_{2}$ in the microspheres. Based on the TGA analysis, the theoretical refractive indices were calculated to be $1.590,1.600,1.622,1.634,1.651$ and 1.685 for samples M0-M5, respectively.

\section{Super-resolution imaging: wide-field mode and white-light illumination.}

The super-resolution imaging configuration is illustrated in Fig. 6a. The semi-immersed microspheres collect the underlying near-field information of the substrate, magnify it and form a magnified virtual image located at a certain position below the surface of the substrate, which can be picked up by a $100 \times$ objective lens (NA $=0.8$, Olympus BX63). When dimethylsilicone is used as the medium to reinforce the imaging contrast, it is less volatile and can easily spread on the substrate compared with liquid solvents such as ethanol or isopropyl alcohol, as shown in the three dimensional image in Fig. 6b. The chip has nano-patterned structures of $60 \mathrm{~nm}$ and $75 \mathrm{~nm}$ gaps between specific stripes with widths of $115 \mathrm{~nm}$ and $145 \mathrm{~nm}$, as indicated by the arrows in the SEM image (Fig. 6c).

According to the Rayleigh equation, $d=0.5 \lambda / \mathrm{NA},{ }^{8}$ in which $\lambda$ is the illumination wavelength and NA is the numerical aperture of the objective lens. The resolution limit is $344 \mathrm{~nm}$ under white-light illumination, which peaked at $\lambda=550 \mathrm{~nm}$ and $\mathrm{NA}=0.8$ for the optical microscopy used here. Obviously, this microscopy cannot resolve the nanoscale surface features of the chip (see Fig. S2, $\mathrm{ESI} \dagger$ ). However, when the dimethyl-silicone semi-immersed microspheres (diameter $=10 \mu \mathrm{m}$ ) are used as a superlens, the imaging resolutions of the microscope are obviously improved. Nonetheless, the pure polymer microspheres M0 $\left(n_{\mathrm{p}}=1.590\right)$ and hybrid microsphere $\mathrm{M} 1\left(n_{\mathrm{p}}=1.600\right)$ still cannot resolve $75 \mathrm{~nm}$ gaps (indicated by the arrows in Fig. $6 \mathrm{~d}$ and e). With the increase in refractive index of the microspheres, $75 \mathrm{~nm}$ gaps between three stripes with a width of $145 \mathrm{~nm}$ can be clearly discerned (Fig. $6 \mathrm{f}$ and $\mathrm{g})$. As the diameter of the nanocomposite microsphere is increased, with the sample M5 as an example, not only can the $75 \mathrm{~nm}$ features be observed, but the imaging windows significantly increase, as shown in Fig. $6 \mathrm{~h}-\mathrm{k}$. The only drawback is that the dimethyl-silicone semi-immersed microspheres still cannot image the texturing structure with $60 \mathrm{~nm}$ gaps.

To further improve the image resolution, cedarwood-oil was used as the immersion medium instead of dimethyl-silicone for observing the chips with $60 \mathrm{~nm}$ gaps between four $115 \mathrm{~nm}$ wide stripes (Fig. 7a). The three-dimensional images of the microspheres semi-immersed in cedarwood-oil are shown in Fig. 7b. All the microspheres M0, M1, M3, and M5 have almost the same diameter of $\sim 13 \mu \mathrm{m}$ and can resolve the $75 \mathrm{~nm}$ gaps between three $145 \mathrm{~nm}$ wide stripes of the chips (Fig. 7c-e), but only the microsphere M5 with the highest refractive index can resolve the $60 \mathrm{~nm}$ gaps of the chip (Fig. 7f). These results further demonstrate that the microspheres with higher refractive index produce higher resolution and imaging quality compared to those with low refractive index microspheres. The reason is explained below.

\section{Super-resolution imaging: confocal mode and laser illumination}

Furthermore, we investigated the imaging behaviour of the microsphere coupled confocal microscopy. As shown in Fig. 8a, the chip has a periodic structure with minimum gaps of $50 \mathrm{~nm}$. This nanotexturing cannot be resolved by confocal microscopy (Olympus OLS4100 LEXT) with a $100 \times$ and NA = 0.95 objective at the laser illumination of $\lambda=405 \mathrm{~nm}$ (Fig. 8b). However, when the dimethyl silicone semi-immersed microspheres M1, M3, and M5 with almost the same diameters of $13.3 \mu \mathrm{m}$ were placed on top of the chips, the $50 \mathrm{~nm}$ gaps can be resolved (Fig. 8c-e). As the refractive index of the microsphere increased from 1.600 to 1.634 and 1.685 for spheres of M1, M3 and M5, the corresponding image magnifications increased from 1.50 to 1.81 and 2.05 , respectively, indicating that 

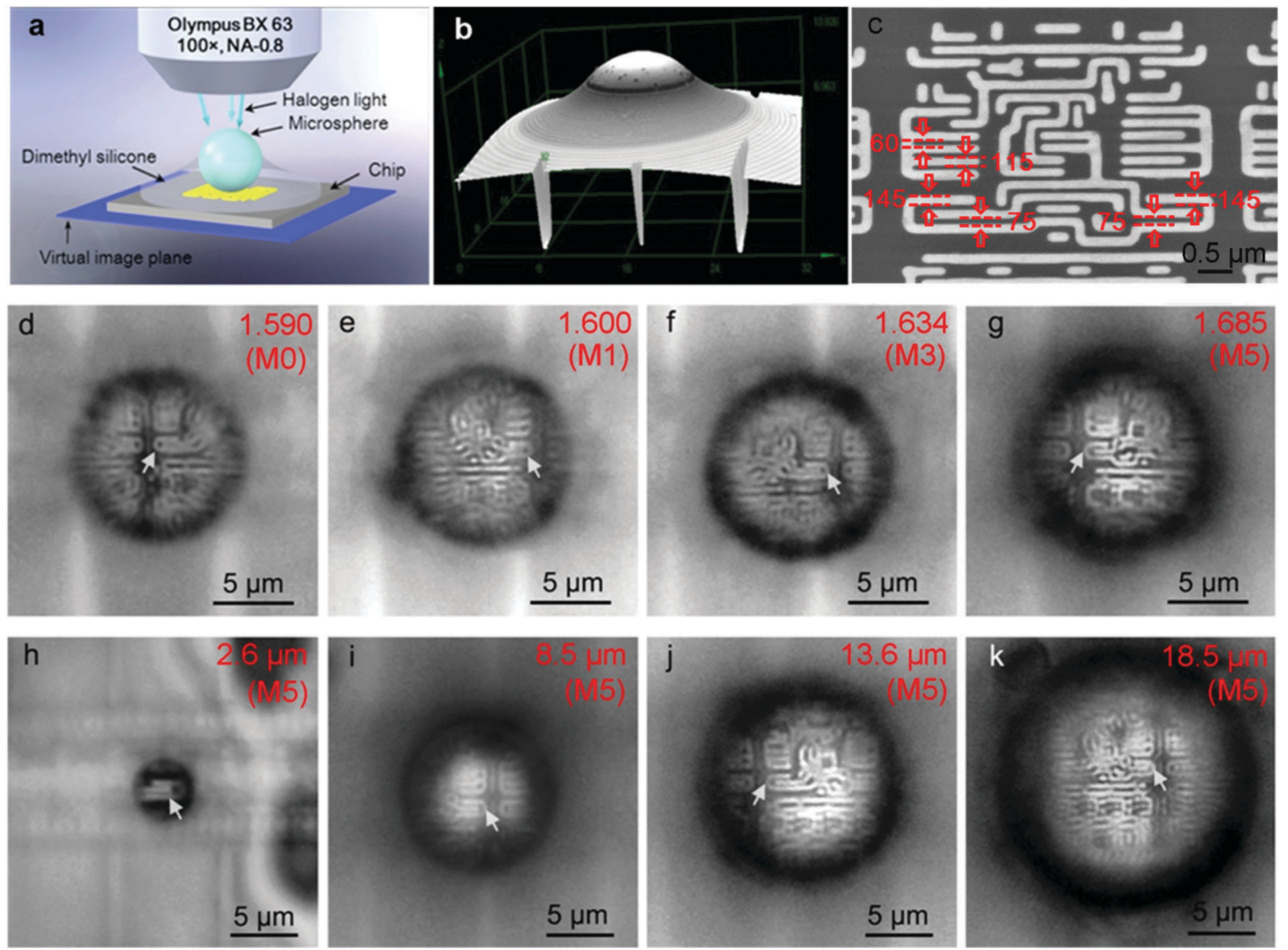

Fig. 6 (a) Schematic of the dimethyl-silicone semi-immersed microsphere for super-resolution imaging under white-light illumination; (b) three dimensional image showing the distribution of dimethyl-silicone around the sphere; (c) SEM image of the nano-patterned chips with $75 \mathrm{~nm}$ and $60 \mathrm{~nm}$ gaps between $145 \mathrm{~nm}$ and $115 \mathrm{~nm}$ wide stripes; $(\mathrm{d}-\mathrm{g})$ optical super-resolution imaging of nano-patterned chips by M0 (1.590), M1 (1.600), M3 (1.634), and M5 (1.685) in the same diameters of $10 \mu \mathrm{m}$; ( $\mathrm{h}-\mathrm{k})$ optical super-resolution imaging of the chips by M5 with different diameters of $2.6 \mu \mathrm{m}, 8.5 \mu \mathrm{m}, 13.6 \mu \mathrm{m}$, and $18.5 \mu \mathrm{m}$, the corresponding imaging magnifications of these pictures are $\times 2.41, \times 2.31, \times 2.10$, and $\times 2.08$, respectively. The white arrows in ( $d-k$ ) indicate the location of the structure containing $75 \mathrm{~nm}$ gaps in the image.

microspheres with higher refractive index can produce better super-resolution imaging.

\section{Super-resolution mechanism}

The basic mechanism of microsphere superlens imaging was explained in our previous study on the technique. ${ }^{2}$ The microspheres function as a superlens, producing sub-wavelength focusing, which illuminates the sample for imaging. Taking the case of cedarwood-oil $\left(n_{\mathrm{m}}=1.51\right)$ as an example (Fig. 7 experiments), Mie theory calculations show that a $13 \mu \mathrm{m}$ microsphere can generate an illumination spot of $\sim 120 \mathrm{~nm}$ (M0), $117 \mathrm{~nm}$ (M1), $112 \mathrm{~nm}$ (M3), $110 \mathrm{~nm}$ (M5) at the sample surface plane, as shown in Fig. 9 (red curve, labelled as bright stripes). All the spheres (M0-M5), therefore, have the capability of resolving $145 \mathrm{~nm}$ strip features; this agrees with our experimental observations (Fig. 7c-f). On the other hand, only M3 and M5 are predicted to be able to see $115 \mathrm{~nm}$ features. Our experiments confirm that M5 can indeed visualize the $115 \mathrm{~nm}$ stripes (Fig. 7f), but M3 failed (Fig. 7e); this could be caused by imperfection in the microspheres and optical systems or wave interference between particle and samples. Once the $115 \mathrm{~nm}$ features are picked up by the M5 lenses (Fig. 7f), they were magnified by 2.1 times (becoming $241.5 \mathrm{~nm}$ ) and projected into the conventional objective lens (NA: 0.8); here, the magnification $\mathrm{M}$ of $\mathrm{M} 5$ lenses in Fig. $7 \mathrm{f}$ was obtained by calculating the ratio of the image size of the stripes and corresponding real size of the stripes. Using the following equation, we can inversely calculate the minimum gap size (i.e., gap resolution $d_{\text {gap }}$ ) between two strips, which is resolvable by the M5 microsphere superlens:

$$
\left(d_{\text {gap }}+d_{\text {strip }}\right) \times M=\lambda / 2 \mathrm{NA}
$$

where $d_{\text {strip }}=115 \mathrm{~nm}$ is the strip size; $M=\sim 2.1$ is the magnification factor; $\lambda=550 \mathrm{~nm}$ and $\mathrm{NA}=0.8$; the gap resolution $d_{\text {gap }}$ was found to be $49 \mathrm{~nm}$. This explains why the M5 superlens can resolve $115 \mathrm{~nm}$ features with $60 \mathrm{~nm}$ gaps. According to our calculation, the gap resolution can go to as low as $20 \mathrm{~nm}$ for $n=1.76$ microspheres, with stripes sized larger than $140 \mathrm{~nm}$. On the other hand, the strip resolution can go to 

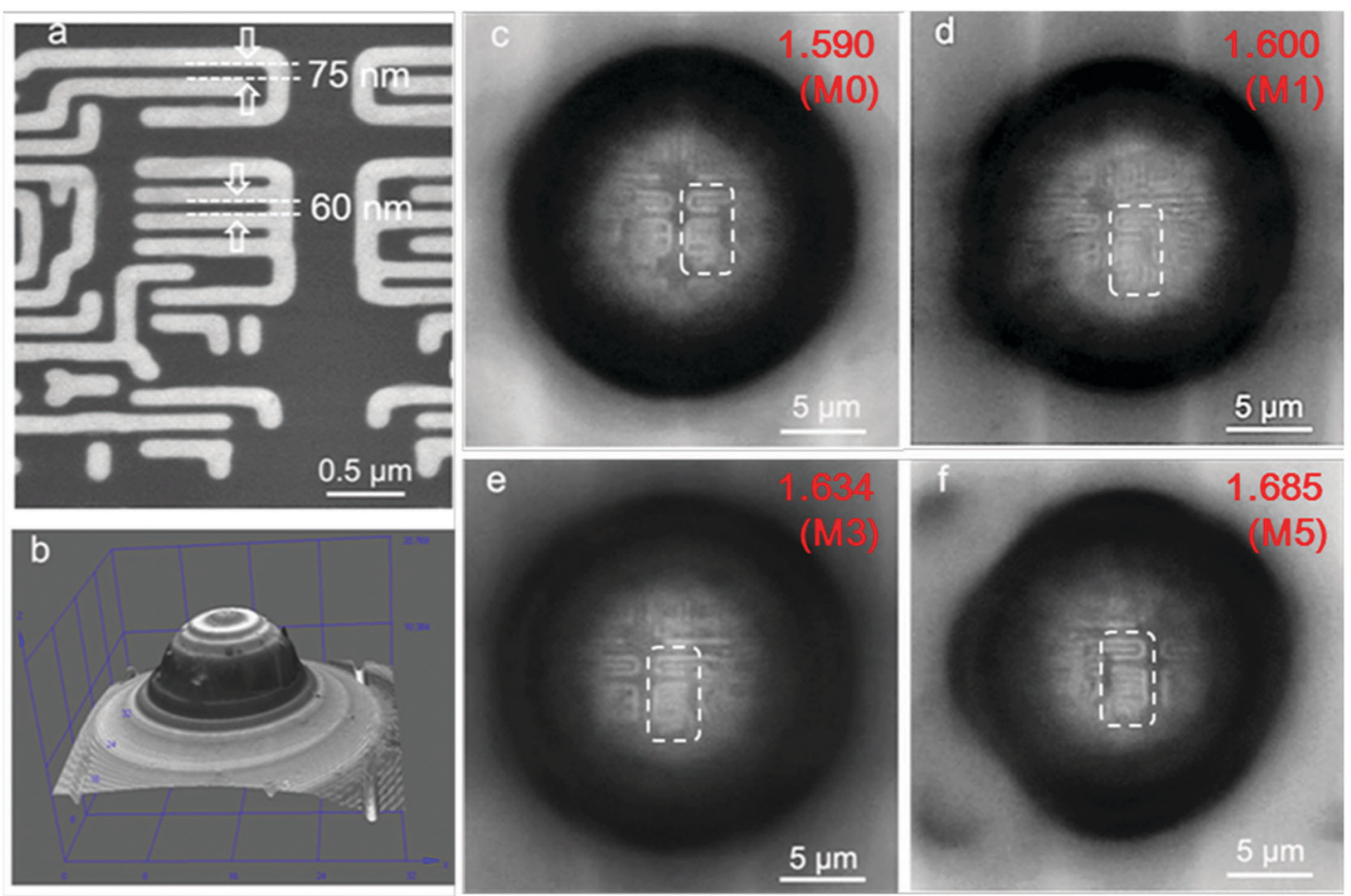

Fig. 7 (a) SEM image of the nano-patterned chips with $60 \mathrm{~nm}$ gaps between four $115 \mathrm{~nm}$ wide stripes; (b) three dimensional image showing the distribution of cedarwood-oil around the sphere; (c to f) optical super-resolution imaging of the chips by M0, M1, M3, and M5, respectively, the dotted bordered rectangles in these pictures indicate locations of the structure containing $75 \mathrm{~nm}$ and $60 \mathrm{~nm}$ gaps between $145 \mathrm{~nm}$ wide stripes (upper three stripes) and $115 \mathrm{~nm}$ stripes (lower four stripes).

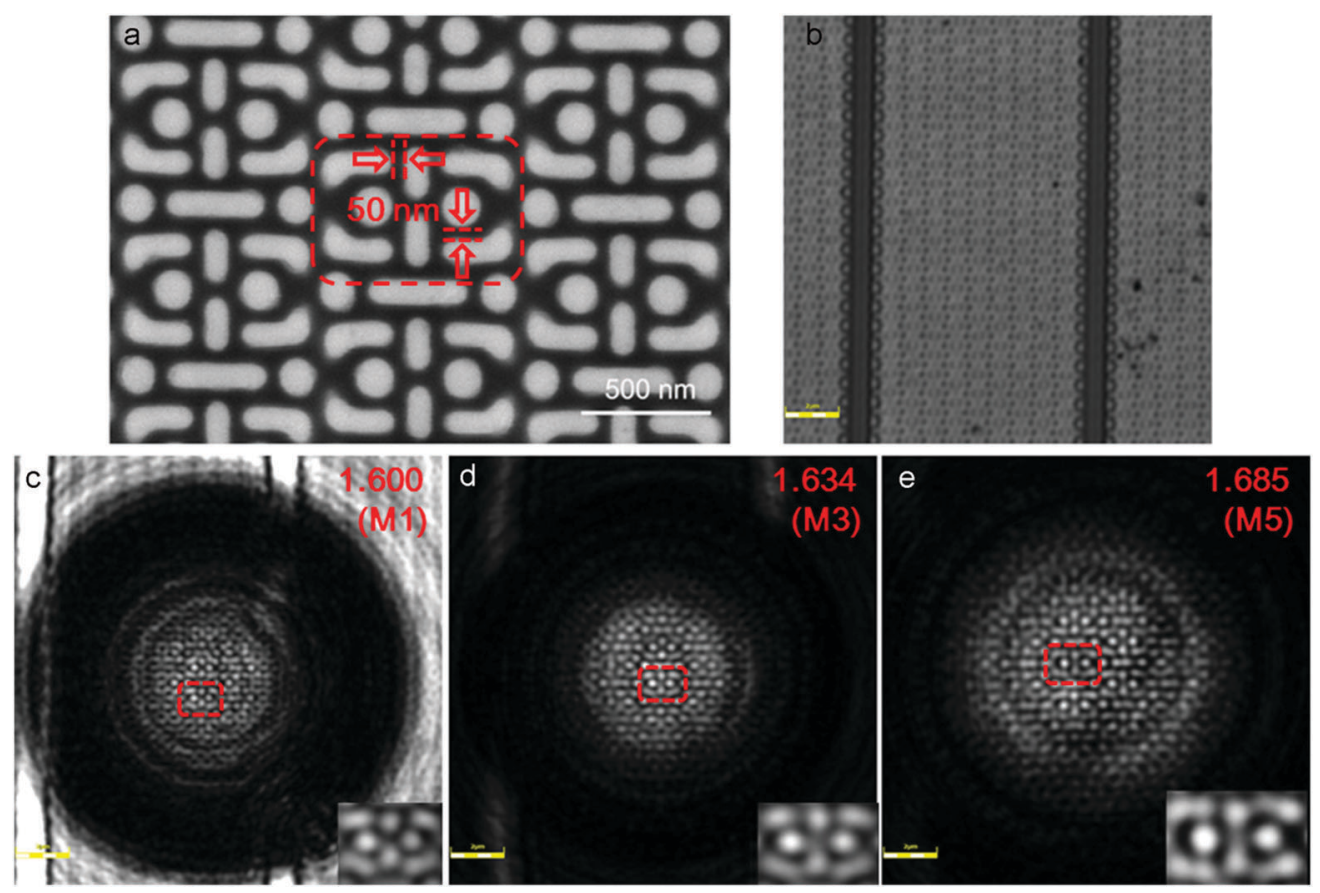

Fig. 8 (a) SEM image of the chip with periodic structures containing $50 \mathrm{~nm}$ gaps; (b) confocal image at $\lambda=405 \mathrm{~nm}$ of the same chip in (a); (c to e) imaging of the chips at $\lambda=405 \mathrm{~nm}$ through $13.3 \mu \mathrm{m}$ spheres of M1, M3, and M5, respectively; the dotted bordered rectangles in these images indicate locations of the structure containing $50 \mathrm{~nm}$ gaps that are resolved by these spheres, the corresponding magnified images are shown in the bottom right corners. The parameters of the objective lens are $100 \times, \mathrm{NA}=0.95$, and the bars are $2 \mu \mathrm{m}$ in $(\mathrm{b}-\mathrm{e})$. 


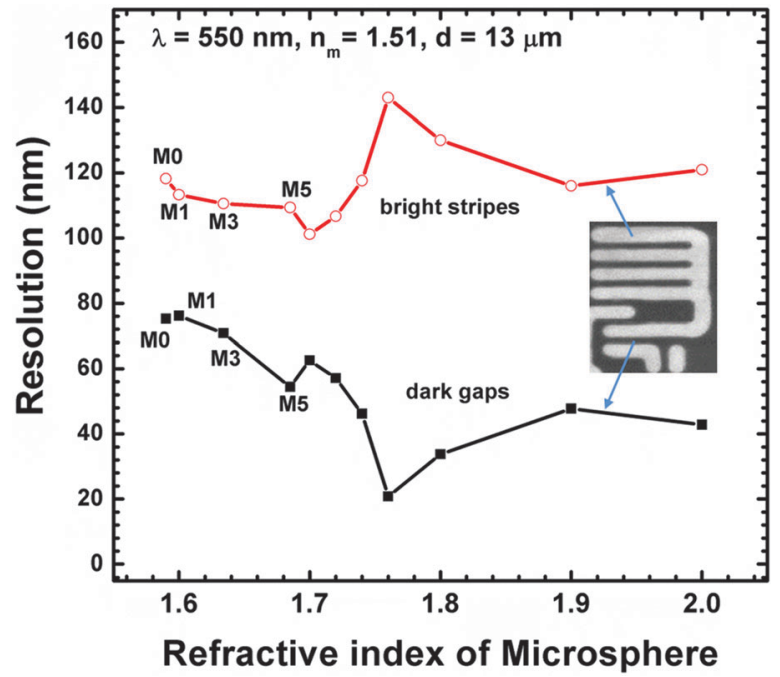

Fig. 9 Resolution (i.e., minimum resolvable features for bright stripes and dark gaps) as a function of refractive index of microspheres calculated with the assistance of Mie theory.

as low as $100 \mathrm{~nm}$ for $n=1.7$ microspheres, with a resolvable gap size of $60 \mathrm{~nm}$.

\section{Conclusions}

Based on this study, a novel type of nanohybrid colloidal microspheres with high refractive index and optical transparency have been successfully synthesized through suspension polymerization of MPS and OA modified $\mathrm{ZrO}_{2}$ nanoparticles with styrene. Because these modified $\mathrm{ZrO}_{2}$ nanoparticles have very good compatibility and reactivity with the monomers, as high as $50.8 \mathrm{wt} \%$ of $\mathrm{ZrO}_{2}$ nanoparticle can be homogeneously dispersed in the hybrid microspheres. When this new class of high refractive index and optically transparent $\mathrm{ZrO}_{2} / \mathrm{PS}$ hybrid microspheres are used as a superlens, the dimethyl-silicone oil semi-immersed microspheres can discern surface features at $75 \mathrm{~nm}$, and the cedarwood-oil semi-immersed microspheres can reach a super-resolution imaging of $60 \mathrm{~nm}$ under white-light microscopy. Under laser illumination at $\lambda=405 \mathrm{~nm}$, these microspheres can even resolve more complicated structures with $50 \mathrm{~nm}$ gaps. The synthesis of hybrid microspheres is feasible, easily repeatable and designable in shape, size and refractive index. Accordingly, the approach that we have described here is quite general, and can be readily extended to prepare a series of hybrid microspheres with various refractive index and optical transparency, by changing the types of polymers and nanoparticles. The as-synthesized nanohybrid colloidal microspheres can be used not only in optical nanoscopes for super-resolution imaging with visible frequency, but also in some potential fields of nanolithography, optical memory storage, and optical nano-sensing.

\section{Acknowledgements}

Financial support was received from the National Natural Science Foundation of China (Grants 51133001 and 21374018), National
"863" Foundation (2013AA031801), Science and Technology Foundation of Ministry of Education of China (20110071130002), and Science and Technology Foundation of Shanghai (13JC1407800). Bing Yan and Zengbo Wang gratefully acknowledge the financial support provided by the Welsh Government and Higher Education Funding Council for Wales through the Sêr Cymru National Research Network in Advanced Engineering and Materials.

\section{Notes and references}

1 H. Yang, N. Moullan, J. Auwerx and M. A. M. Gijs, Small, 2014, 10, 1712-1718.

2 Z. Wang, W. Guo, L. Li, B. Luk'yanchuk, A. Khan, Z. Liu, Z. Chen and M. Hong, Nat. Commun., 2011, 2, 218.

3 E. Betzig, G. H. Patterson, R. Sougrat, O. W. Lindwasser, S. Olenych, J. S. Bonifacino, M. W. Davidson, J. L. Schwartz and H. F. Hess, Science, 2006, 313, 1642-1645.

4 N. Fang, H. Lee, C. Sun and X. Zhang, Science, 2005, 308, 534-537.

5 E. T. Rogers, J. Lindberg, T. Roy, S. Savo, J. E. Chad, M. R. Dennis and N. I. Zheludev, Nat. Mater., 2012, 11, 432-435.

6 I. I. Smolyaninov, Y. J. Hung and C. C. Davis, Science, 2007, 315, 1699-1700.

7 D. Kang, C. Pang, S. M. Kim, H. S. Cho, H. S. Um, Y. W. Choi and K. Y. Suh, Adv. Mater., 2012, 24, 1709-1715.

8 J. Y. Lee, B. H. Hong, W. Y. Kim, S. K. Min, Y. Kim, M. V. Jouravlev, R. Bose, K. S. Kim, I.-C. Hwang and L. J. Kaufman, Nature, 2009, 460, 498-501.

9 A. Vlad, I. Huynen and S. Melinte, Nanotechnology, 2012, 23, 285708.

10 D. Wu, S.-Z. Wu, L.-G. Niu, Q.-D. Chen, R. Wang, J.-F. Song, H.-H. Fang and H.-B. Sun, Appl. Phys. Lett., 2010, 97, 031109.

11 Y.-L. Sun, W.-F. Dong, R.-Z. Yang, X. Meng, L. Zhang, Q.-D. Chen and H.-B. Sun, Angew. Chem., Int. Ed., 2012, 51, 1558-1562.

12 A. Darafsheh, G. F. Walsh, L. Dal Negro and V. N. Astratov, Appl. Phys. Lett., 2012, 101, 141128.

13 S. Lee, L. Li, Y. Ben-Aryeh, Z. Wang and W. Guo, J. Opt., 2013, 15, 125710.

14 L. Li, W. Guo, Y. Yan, S. Lee and T. Wang, Light: Sci. Appl., 2013, 2, e104.

15 X. Hao, C. Kuang, X. Liu, H. Zhang and Y. Li, Appl. Phys. Lett., 2011, 99, 203102.

16 R. Ye, Y. H. Ye, H. F. Ma, L. Cao, J. Ma, F. Wyrowski, R. Shi and J. Y. Zhang, Sci. Rep., 2014, 4, 3769.

17 A. Darafsheh, N. I. Limberopoulos, J. S. Derov, D. E. Walker and V. N. Astratov, Appl. Phys. Lett., 2014, 104 061117.

18 C. L. Lü and B. Yang, J. Mater. Chem., 2009, 19, 2884-2901.

19 S. Lee, H.-J. Shin, S.-M. Yoon, D. K. Yi, J.-Y. Choi and U. Paik, J. Mater. Chem., 2008, 18, 1751-1755. 
20 C. L. Lü, C. Guan, Y. F. Liu, Y. R. Cheng and B. Yang, Chem. Mater., 2005, 17, 2448-2454.

21 M. N. Tchoul, S. P. Fillery, H. Koerner, L. F. Drummy, F. T. Oyerokun, P. A. Mirau, M. F. Durstock and R. A. Vaia, Chem. Mater., 2010, 22, 1749-1759.

22 C. Lü, Y. Cheng, Y. Liu, F. Liu and B. Yang, Adv. Mater., 2006, 18, 1188-1192.
23 G. Garnweitner, L. M. Goldenberg, O. V. Sakhno, M. Antonietti, M. Niederberger and J. Stumpe, Small, 2007, 3, 1626-1632.

24 S. X. Zhou, G. Garnweitner, M. Niederberger and M. Antonietti, Langmuir, 2007, 23, 9178-9187.

25 S. X. Zhou and L. M. Wu, Macromol. Chem. Phys., 2008, 209, 1170-1181. 